\title{
Tensiones entre 'asistencia territorializada' y 'capacitación para la empleabilidad' en espacios rurales: el caso de los jóvenes de Centro del Capacitación para el trabajo de Costa de Araujo, Mendoza (Argentina)
}

\author{
Diego Quattrini* y Carla Daniela Rosales*
}

\author{
Categoría: Estudio \\ Fecha de recepción: 23 de mayo de 2012 \\ Fecha de aprobación: 27 de junio de 2012
}

\begin{abstract}
Resumen
Se propone un análisis sobre las políticas educativas y de inserción social destinadas a los y las jóvenes incluidos en un espacio neo-colonial, agrario y periférico. Para lograr este objetivo fin se toma el distrito de Costa de Araujo, ubicado en Lavalle, provincia de Mendoza (Argentina), siendo este un territorio marginal que ha sufrido las transformaciones de reestructuración capitalista en el agro. En este contexto, se estudia el papel creciente que ha asumido el sistema educativo en la implementación de políticas de formación para jóvenes rurales. Por ello, se analiza el único centro de Capacitación para el Trabajo de la zona y su propuesta institucional, basada en: la terminalidad educativa secundaria y la capacitación de la fuerza de trabajo, joven en este caso. Por lo referido, se reflexionará acerca de esta propuesta educativa a la luz de la realidad social de la juventud rural en Costa de Araujo.
\end{abstract}

Palabras claves: juventud rural, educación, capacitación, capitalismo e inclusión

Abstract:

This paper proposes an analysis on educational policies and social integration to the young people belonging to a neo-colonial, agrarian and peripheral configuration. For this purpose we study the case of the district of Costa de Araujo, located in the province of Mendoza

\footnotetext{
* Licenciado en Sociología. Doctorando en Ciencias Sociales - Facultad de Ciencias Políticas y Sociales - Universidad Nacional de Cuyo (Argentina) - Becario de CONICET - CCT- Mendoza-INCIHUSA. Miembro del Grupo de Investigación sobre ‘Conflictividad social en la Provincia de Mendoza'. Correo: dquattrini@mendoza-conicet.gov.ar

* Licenciada y Profesora en Trabajo Social. Doctoranda en Estudios Sociales AgrariosCentro de Estudios Avanzados (CEA)-Universidad Nacional de Córdoba- Becaria de CONICET -CCT- Mendoza-INCIHUSA. Integrante del proyecto PICT N $\mathrm{N}^{\mathrm{o}}$ 01466: "Relacionesespacio-sociedady naturaleza-cultura. Territorioy Desarrolloregionalentierras secas del centro-oeste y noroeste de argentina". E-mail: crosales@mendoza-conicet.gov.ar
} 
Tensiones entre ‘asistencia territorializada〉 y «capacitación para la empleabilidad en espacios rurales: el caso de los jóvenes de Centro del Capacitación para el trabajo de Costa de Araujo, Mendoza (Argentina) - Diego Quattrini y Carla Rosales

(Argentina), which is characterized by a marginal area that has undergone The current transformations of agrarian capitalist structures. To achieve the objective, we examines the increasing role that have assumed the educational system on the implementation of policies for training for rural youth. In this sense, we present an analysis of the only training center for the work in the selected area and its proposal that contains as institutional goals: offer the posibilitiy to finish secondary education and train the workforce of young people of the place. This approach is intended to reflect on these educational proposals in the light of the particular social reality that assumes rural youth in Costa de Araujo.

Keywords: rural youth, education, training, capitalism and inclusion

\section{Introducción}

Acá están cansados por el trabajo [] porque no les gusta, porque van a trabajar y ganan plata... es decir siempre son bastantes recios [] acá [] el que tiene 16, 17 años acá es un hombre [] porque tiene que salir a trabajar al lado de su padre [] tiene que hacer la misma tarea. (Director de un Colegio Primario de Jóvenes y Adultos, Ceba, Costa de Araujo)

Existen en ciencias sociales escasos estudios sobre jóvenes rurales que analicen las problemática relación entre el binomio educación - trabajo en tiempos de reestructuración capitalista. A pesar de aumentar notablemente el número de trabajos que investigan las temáticas juveniles, quienes abordan el concepto de juventud lo hacen de una manera abstracta al presentarlo en cierta medida bajo un cierto sesgo "urbano modernizante" -son jóvenes que poseen una "moratoria social" (Margulis, 2001)- y para quienes trabajan más específicamente la "juventud rural" o a los "jóvenes campesinos", las producciones sólo se limitan a analizar su abordaje asociada a la cuestión de la migración del campo a la ciudad y a la sucesión de la pequeña propiedad de la tierra (De Castro, 2009:187). En otro orden, la mayoría de los intelectuales que suscriben la tesis de la nueva cuestión rural, estarían de acuerdo hoy, que ante los procesos de segregación económica y social que afectaron al sector agrícola latinoamericano, 
los jóvenes deben hacer frente a una situación laboral y social diferente que la de su generación precedente. En este marco para comenzar a ordenar el estudio es preciso partir un análisis que incorpore como precisa la importancia de la condición y posición específica que poseen estas poblaciones dentro de la estructura societal actual. En una primera mirada y partiendo de las declaraciones del director de la escuela primaria para adultos de Costa de Araujo, la situación social de estos adolescentes posee una cierta particularidad: "deben ya hacerse hombres y salir a trabajar tempranamente a la par de sus mayores", dificultando su desarrollo formativo y educacional. Esta "posición y condición" de la juventud rural juvenil queda al parecer marcada por una serie de procesos que se discutirán en adelante: una posición dentro de la unidad doméstica particular; un significado, una obligación y dificultades de "ir a trabajar"; y oportunidades y limitaciones que presenta el "ir a estudiar" en estos espacios.

Pero para avanzar en la indagación de las propuestas de educación y formación para el trabajo para estos jóvenes, las mismas deben ser leídas en primera instancia a la par del análisis de las transformaciones sucedidas en los espacios agrarios capitalistas periféricos. Pues, aquí la desposesión y mercantilización continua de los bienes comunes (preferentemente la monopolización de la tierra y del agua) se presenta como un rasgo estructurante que marca la constitución de las formas de expropiación que podríamos llamar capitalismo neo-colonial. Las relaciones sociales de producción en esta fase de acumulación se sustentan en la extensión de una sustracción y depredación sistemática de nuevos activos naturales y sociales que realizan unos pocos grupos concentrados, los cuales disponen de grandes volúmenes de poder. Siguiendo los planteos de Scribano (2010) con la idea de "actitud neo-colonial" y depredación de las condiciones biológicas y materiales no sólo incluimos lo que usualmente entendemos como bienes comunes, sino además la extracción de todas las fuentes de energía en diferentes territorios, tanto las de los recursos ambientales como de las energías 
Tensiones entre ‘asistencia territorializada) y (capacitación para la empleabilidad en espacios rurales: el caso de los jóvenes de Centro del Capacitación para el trabajo de Costa de Araujo, Mendoza (Argentina) - Diego Quattrini y Carla Rosales

corporales necesarias para mantener la plusvalía salarial y la reproducción de la fuerza de trabajo.

La "actitud neocolonial" queda plasmada cuando se observan ciertos procesos sociales que experimenta el mundo rural y que en varios estudios sociales dan cuenta: una mayor concentración de la tierra, las dificultades del pequeño productor de mantenerse en sus chacras y de generar alimentos para el autoconsumo, la necesidad de intensificar la asalarización parcial o total de los integrantes de las familias rurales (Neiman 2006:40); la resignificación y refundización de la precarización del trabajo rural (como el bajo salario y su pobre protección social) (Bendini, Radonich, Steimbreger, 2007:81), etc. Sin embargo, esta actitud, también puede ser observada en los nuevos dispositivos puestos en juego que se despliegan para preparar las sociabilidades de los jóvenes - trabajadores. Para que la reproducción siga su curso, en lo cotidiano, es necesario activar un conjunto de instituciones que promuevan ciertas prácticas sociales que trabajen sobre las sensibilidades juveniles. Practicas que por un lado hagan "soportable la vida" y por ostro estructuren modos socialmente diferenciados de "percibir y percibirse", a fin de suturar las posibles contradicciones y grietas que pueda aparecer en el proceso de aceptación social de la dominación ${ }^{1}$ (Scribano, 2007). En esta lí-

1 Aquí nos referimos a la extensión de los mecanismos de soportabilidad social y los dispositivos de regulación de sensaciones que posee como características el régimen neo-colonial (Scribano, 2007). Los "mecanismos de soportabilidad social" constituyen una suerte de habitus en el sentido bourderiano -estructura, estructurada, estructurante de prácticas- encargadas de evitar los conflictos sociales. Son parte de las muchas prácticas cotidianas, incorporadas a lo largo de la socialización. Estas particularmente se encargan de hacer soportable la vida, a partir de la naturalización, neutralización, oclusión, desplazamiento o inversión de las situaciones conflictivas. Estos mecanismos se articulan con los "dispositivos de regulación de las sensaciones" que constituyen modos de percibir y percibirse en los juegos de relaciones intersubjetivas en los que los sujetos se hallan inmersos. Las percepciones -definidas como esquemas de clasificación, apreciación y anticipación de los cuerpos ubicados en un espacio-tiempo sociales determinados- conforman di-visiones del mundo, visiones que diferencian muchas veces a partir de opuestos, que permiten anticipar las prácticas. 
nea de análisis nuestra hipótesis señala que las políticas públicas educativas aparecen como un engranaje en la formación de mecanismos de la dominación trabajando diferencialmente sobre las distintas poblaciones y clases sociales, es decir interviniendo en un marco de "asistencia territorializada" (Álvarez Leguizamón 2011) en que, como foco de atención, el sector de los jóvenes rurales no presentan la excepción. Esta hipótesis señala más específicamente, que estas políticas han sido implementadas con el objetivo, por un lado, de frenar el deterioro de las condiciones de vida y la exclusión social (de contener e incluir), y por otro de formar subjetividades para la producción y para las exigencias de un mercado laboral regional flexibilizado (de preparar la fuerza juvenil de trabajo).

Al respecto resulta emblemático el papel creciente que han alcanzado los Centros de Capacitación para el Trabajo (CCT), convirtiéndose en medios alternativos para reinsertar en los sistemas formales a aquellas poblaciones vulnerables que presentan rezagos escolares a través de su propuesta de capacitación en oficios. De esta manera, y teniendo en cuenta la impronta de los CCT en el campo social, para el abordaje del estudio de las políticas de educación y formación para el empleo y su vinculación con la dominación se realizará un análisis sobre el centro de Capacitación para el Trabajo en el distrito de Costa de Araujo, en el departamento de Lavalle (en la provincia de Mendoza).

El trabajo está organizado principalmente en dos secciones. En una primera instancia discutiremos la noción de juventud rural en relación con la abstracción que muchos pensadores realizan de la categoría juventud. Luego se realizará una aproximación a las características que presenta el mercado de trabajo rural. Aquí cobran importancia las transformaciones agrarias particulares y recientes, a partir de las cuales se han reconfigurado los espacios regionales y se han reactualizado viejas y nuevas modalidades de precarización del empleo. Junto con el análisis del impacto de estas mutaciones se propone un acercamiento a las situaciones y 
Tensiones entre ‘asistencia territorializada) y ‘capacitación para la empleabilidad en espacios rurales: el caso de los jóvenes de Centro del Capacitación para el trabajo de Costa de Araujo, Mendoza (Argentina) - Diego Quattrini y Carla Rosales

las condiciones educativas de jóvenes del sector rural de Costa de Araujo.

En una segunda instancia se aborda el análisis del caso de la Escuela de Capacitación para el Trabajo de Costa de Araujo. Nuestro planteo es que la vigencia de estos centros son el resultado de la continuidad del paradigma neoliberal en el campo de las políticas públicas educativas originadas en los '90. En esta línea se explora la propuesta educativa que actualmente ofrece el CCT en base a dos metas institucionales, por un lado la terminalidad educativa y por otro la capacitación de la fuerza de trabajo. El objetivo será realizar una aproximación al análisis de la relación entre configuración de sociabilidades - modos de educación modos de trabajo.

En relación a la metodología, las reflexiones del estudio provienen preferentemente desde los aportes de la sociología rural, la sociología del cuerpo y las emociones y la sociología de la juventud. A partir de ciertas reflexiones se decidió encarar un diseño metodológico más ligado a la tradición cualitativa, en la medida que permitió acercarnos a la problemática de los modos de construcción de sociabilidades en el espacio de la juventud rural. Con el uso de las técnicas cualitativas se pretendió construir datos de una manera flexible priorizando la opinión de los sujetos que protagonizan las prácticas seleccionadas en el estudio. Elegimos realizar un estudio de caso, es decir, analizar el Centro de Capacitación para el Trabajo de Costa de Araujo (el único de la zona) porque el mismo adquiere por un lado un papel importante en la comunidad y por otro condensa de manera significativa en sus objetivos las metas institucionales de las políticas públicas formales educativas.

Más específicamente, para acercarnos al análisis del funcionamiento institucional se realizaron entrevistas a su directora y docentes del centro de capacitación. Además se realizó una observación de campo del funcionamiento cotidiano institucional y se registró documentación específica sobre la oferta educativa. 
Para completar la información se trabajó en la implementación de un grupo focal con alumnas y ex-alumnas de los trayectos educativos. Finalmente en el marco de investigaciones de tesis doctoral se realizaron diferentes entrevistas a otros pobladores de Costa de Araujo con la intención de comprender algunas características de esta comunidad y aproximarse a la "mirada social" que posee el centro educativo.

\section{Hacia una aproximación de la noción de Juventud rural}

El concepto de juventud en la literatura de las Ciencias Sociales posee, en general, una carga teórica 'modernizante', 'urbana' y 'capitalista', cuando se la comienza a tratar como una categoría histórica y no como un mero hecho biológico. Sin duda, la Revolución Industrial tuvo mucho que ver con todo ello. Con un cierto tono metafórico Frank Musgrove (1965:33) ha afirmado que el joven fue inventado al mismo tiempo que la máquina a vapor. $\mathrm{Si}$ bien dicha máquina fue inventada por Watt en 1765, el del joven fue Rousseau, en 1762. Este pensador moderno descubre el reino de la adolescencia y su insistencia en el carácter natural de esta fase de la vida, para él la inevitabilidad de sus crisis, la necesidad de segregar a los jóvenes del mundo de los adultos, tendría gran influencia en las teorías posteriores de los psicólogos y pedagogos (Feixá, 1999:33)

Con relación al subimiento moderno de la juventud, será entrado el siglo XX cuando emerja desde la psicología la noción de moratoria social. La misma alude a un plazo concedido a cierta clase social de jóvenes, que les permite gozar de una menor exigencia mientras completan su instrucción y alcanzar su madurez social y económica (Margulis, 2001:43).

Este periodo destinado principalmente a la preparación, hace referencia a la condición de estudiante (principalmente masculina) y se remite a una clase media (urbana) que puede postergar 
Tensiones entre ‘asistencia territorializada〉 y «capacitación para la empleabilidad en espacios rurales: el caso de los jóvenes de Centro del Capacitación para el trabajo de Costa de Araujo, Mendoza (Argentina) - Diego Quattrini y Carla Rosales

tanto el matrimonio como su inserción económica en pos de prolongar su formación universitaria (Margulis, 2001). Esta noción se ha ido asociando a una visión implícita de la juventud como fenómeno universal, pero que ha sido fuertemente criticada por su aplicabilidad para todos los jóvenes por igual. De esta manera $\mathrm{y}$ ante las evidentes transformaciones del siglo $\mathrm{XX}$, tales como la expansión del sistema educativo, cambios en el mercado de trabajo, nuevas relaciones inter e intrageneracionales, relaciones que muestran nuevas formas de habitar la juventud, resulta indispensable la necesidad de romper con visiones universalistas y homogeneizadoras.

Superar la noción de moratoria social aplicada a las juventudes rurales deviene en superar el mandato erróneo de que en el campo no existe la juventud, pues esta contiene otras características y particularidades que la definen como tal. Actualmente, han desaparecido los ritos de pasaje de una etapa vital a otra y se ha reducido la predictibilidad respecto de los lugares sociales que ocupará cada sector etario. Hay distintas maneras de ser joven en el marco de la intensa heterogeneidad que se observa en el plano económico, social y cultural (Margulis, 2001:42). Asimismo podemos distinguir múltiples factores que van configurando también los espacios vivenciales de la juventud rural, tales como: la condición de género, la generación, la pertenencia étnica, la situación geográfica y el estrato socioeconómico de los individuos.

Sin embargo nos encontramos ante una escasez de estudios sobre las condiciones de la juventud rural. Esta invisibilidad teórica y política se ve reforzada por, al menos, tres factores: alta concentración de jóvenes en las ciudades (escaso peso demográfico, cerca del $8 \%$ en zonas rurales); el sesgo urbanizante y modernizante del concepto de juventud, pues subyace en él la idea de progresiva extinción de los espacios rurales; y el hecho de que la juventud rural no se presenta en la sociedad como un "problema social" tan visible como en el caso de sus pares urbanos, quienes son juzgados más fuertemente por sus adicciones, embarazos 
adolescentes, por sus índices de delincuencia, entre otros. En tal sentido, Luis Caputo afirma que tampoco existen instrumentos estadísticos aplicados para conocer el nivel de oportunidades que tienen las comunidades rurales (Caputo, 2002).

Entre los pocos estudios, el análisis de Kessler menciona que la particularidad de la juventud rural estaría dada por sus relaciones familiares patriarcales, que existiría en ella una tendencia hacia la pluriactividad como una forma de subsistencia familiar y que su condición territorial se debatiría entre permanecer y migrar (Kessler, 2005:12).

En el caso brasilero, Elisa Guaraná de Castro afirma que existen factores que afectan particularmente a los jóvenes rurales, de allí que sea el conocimiento de las unidades domésticas el que contribuya a comprender ciertas dinámicas que condicionan sus futuras trayectorias. Entre los elementos que la autora destaca está el peso de la autoridad paterna:

las percepciones de la juventud están marcadas por la construcción de que ese joven debe ser vigilado y controlado. El peso de la autoridad paterna en el espacio doméstico es reproducido en las relaciones de trabajo familiar y en la organización de la explotación. Esa autoridad crea mecanismos de vigilancia y control a través de las relaciones familiares y demás redes sociales, principalmente en las mujeres que se extienden en los espacios que frecuentan (De Castro, 2009:193; traducción propia).

En este sentido, el resultado de la relación jerárquica entre adolescentes y adultos, perfila una construcción específica de la categoría "joven rural", pues será la búsqueda de autonomía (pretensión de alejarse de esa autoridad) el motor que definirá ciertos rasgos de la identidad de algunos de ellos. En este sentido Guaraná de Castro concluye su afirmación:

Juventud/joven asociada a la transitoriedad del ciclo de la vida o biológico, transfiere para aquellos que son así 
Tensiones entre 〈asistencia territorializada〉 y «capacitación para la empleabilidad en espacios rurales: el caso de los jóvenes de Centro del Capacitación para el trabajo de Costa de Araujo, Mendoza (Argentina) - Diego Quattrini y Carla Rosales

identificados, la imagen de individuos o grupos de individuos que necesitan ser regulados, controlados, encaminados. Juventud rural es una categoría especialmente reveladora de esa construcción de jerarquía social. El análisis de esa categoría permite percibir como los procesos de construcción de categorías sociales configuran y refuerzan relaciones de jerarquía social (De Castro, 2009:195, traducción propia).

Además de algunas particularidades de la juventud rural, es necesario entenderla también y en constante intercambio con la ciudad, debido al proceso fuerte de transformación del capitalismo agrario. En este marco las diversas esferas de la vida rural se verán alteradas, cobrando así un nuevo significado los diferentes ámbitos de la educación y del trabajo.

\section{Problemáticas de las situaciones educativas y laborales de los jóvenes rurales}

Comenzando con el análisis de la situación del empleo rural, mencionaremos algunas características que ha adquirido el mercado laboral, en este caso periférico del capitalismo agrario, resultado de las transformaciones de la década de los ‘90.

Siguiendo la aproximación topológica de los agentes sociales en el agro reestructurado propuesta por Murmis (2004), en la cual identifica complejos agroindustriales, campesinos, asalariados rurales, nos ocuparemos de estos últimos, dado que la población juvenil de Costa se emplea principalmente como obreros rurales estacionales.

El autor menciona como un fenómeno que caracteriza al empleo rural en América Latina, a la expansión de la fuerza de trabajo rural asalariado sin tierra, ahora urbana o periurbana, disminuyendo también la importancia relativa del trabajo semiproletario campesino (Murmis, 2004:53). Estas formas modernas de producción siguen demandando trabajo estacional $\mathrm{u}$ ocasio- 
nal, pero profundizando el nivel de inestabilidad, flexibilidad y versatilidad laboral. Esto se traduce para los trabajadores rurales en una explícita y normativa precarización laboral en que los jóvenes son el grupo más perjudicado (Rodríguez y Dabezies, 1991).

En el caso de los jóvenes rurales algunos estudios muestran una particular heterogeneidad en la inserción laboral en los jóvenes. Se identifica para este grupo una importante tasa de trabajo familiar no remunerado; la proletarización rural completa o parcial combinada con trabajo no remunerado en las fincas familiares y ocupaciones informales no rurales; trabajo no agrario de tiempo completo en el sector informal; y un grupo minoritario (real o potencialmente) inserto en sectores dinámicos del rubro servicios o de la producción agroalimentaria (Kessler, 2005). Otros estudios presentados afirman que la juventud rural tiene un contacto más próximo y temprano con el mundo del trabajo, respecto de sus pares urbanos (Durston, 1998).

En cuanto a su situación en el mercado de trabajo, se pueden observar ciertos cambios. Como componentes generales del escenario existen ahora una mayor estacionalidad en la contratación de las tareas rurales, mayores requerimientos de competencias, una profundización en los cambios tecnológicos, entre otros (Neiman 2006: 44). Por ejemplo para el caso mendocino, Bocco (2007) afirma que el cambio tecnológico en la vitivinicultura habría expulsado mano de obra (temporal) y que la mecanización de la producción ha comenzado a requerir obreros más califica$\mathrm{dos}^{2}$. Asimismo, conviven dos formas visibles de acceso al trabajo, sin alejarse de la estacionalidad, por un lado el empleo en las agroindustrias, con contratos formales, y por otro el trabajo en las chacras o fincas, mucho más inestable, pero al menos, este últi-

2 Según datos de nuestras entrevistas, en Costa de Araujo durante la recolección de la aceituna se ha comenzado a demandar operadores calificados para el manejo de máquinas cosechadoras. 
Tensiones entre 〈asistencia territorializada〉 y «capacitación para la empleabilidad en espacios rurales: el caso de los jóvenes de Centro del Capacitación para el trabajo de Costa de Araujo, Mendoza (Argentina) - Diego Quattrini y Carla Rosales

mo, en el caso de los jóvenes que trabajan les permite combinarlo con el estudio vespertino. Una entrevistada comentaba:

[] mi hermana y yo trabajábamos en la chacra [] cuando ella cumplió 18 entró al Molto [] en cambio yo trabajaba más cuando yo iba a la finca a podar o a atar, o si no a cosechar [] a cosechar tomate[] todo eso [] entonces con eso me pagaba las cartillas y si no mi viejo me ayudaba [] cuando sabía que no había trabajo me ayudaba... porque sabía que no podía trabajar [] Y ella no, como siempre ella trabajó en Molto, ella siempre tuvo plata para eso. (Joven entrevistada)

Las nuevas modalidades de empleo no eliminaron las diversas formas de precarización de la fuerza de trabajo como la eventualidad contractual o las formas de pago por destajo, por tarea o por producto. Más bien estos modos de organización del trabajo se reprodujeron y mezclaron con rasgos antiguos de la vulnerabilidad rural: aumento del desplazamiento múltiple de los trabajadores, autoexplotación y diversas modalidades del trabajo "esclavo" (Bendini, et al. 2007). La movilidad territorial que caracterizó al trabajo rural-estacional cobra un significado en el marco de la modernización del agro, pues devienen de este proceso cambios en la relaciones sociales y en los estilos de vida de las comunidades. Como parte de las áreas marginales, los jóvenes se constituyen en reservorios de mano de obra o de trabajo flotante. En coincidencia con la antigua vulnerabilidad rural, una joven comentaba:

cuando llegamos vivíamos en el campamento [] alquilábamos primero [] pero lo que pasa es que si vos vivís ahí tenés que trabajar para el dueño de la tierra [] y él trabajaba más en la temporada en la cosecha y después en la poda [] pero después de ahí no teníamos nada que hacer [] en octubre noviembre no había nada [] en invierno [] entonces mi papá empezó a trabajar en otro lado y entonces como que ya que al hombre ya no le empezó 
a gustar eso y empezó a cobrar caro el alquiler (Joven entrevistada).

A propósito de estas nuevas relaciones de trabajo, una docente afirmaba:

[] la otra empresa grande que siempre ha existido es Cartellone, que ahora no es más Cartellone [] porque incluso ha estado cerrando, ha cambiado de dueño, ha renovado el personal [] ahora están trabajando con personal más temporario... los que eran los más antiguos los han ido liquidando [] cosa que ahora cada tres meses los contratan... todo ese sistema [] son las nuevas tendencias (Docente entrevistada).

En el caso de la situación educativa de los jóvenes rurales, Luis Caputo (2002) en su estudio sobre jóvenes rurales de la Argentina, detectó como dato significativo que un 30\% de los y las jóvenes se encuentran fuera del sistema educativo y del mercado laboral.

Para Elisa Cragnolino (2000) el abandono y la permanencia del sistema educativo queda vinculada directamente con las decisiones de la unidad doméstica familiar. Según su análisis, el ingreso y permanencia de los niños en el sistema escolar está condicionado por el valor asignado a la educación por parte de la familia, como por la necesidad de la fuerza de trabajo infantil y las oportunidades de trabajo existentes en el medio ${ }^{3}$. Es por esto que a la hora de desarrollar algún análisis sobre la educación de los jóvenes rurales es necesario relacionarlo no sólo con las características de la oferta educativa existente sino también con la posición de clase de la familia rural y con las estrategias de

3 Se reconoce la existencia de diferentes modos de trabajo infantil en las zonas rurales. Se distinguen las "actividades centrales", que forman parte principal de la rutina diaria del niño y representan una contribución significativa para el ingreso familiar, de las "actividades auxiliares" que son tareas esporádicas que se combinan con la asistencia a clases y otras actividades del hogar. 
Tensiones entre ‘asistencia territorializada) y ‘capacitación para la empleabilidad en espacios rurales: el caso de los jóvenes de Centro del Capacitación para el trabajo de Costa de Araujo, Mendoza (Argentina) - Diego Quattrini y Carla Rosales

reproducción social que se van configurando en este grupo como unidad (Cragnolino, 2000).

En el caso de nuestra zona de estudio, el ámbito rural de Lavalle, siguiendo el cuadro $\mathrm{N}^{\circ} 1$, si se observa la franja de jóvenes de 13 a 17 años (que abarca el secundario) que "no asiste pero asistió" a la escuela, es decir que han desertado del sistema, aparece una brecha importante entre los chicos de zonas urbanas $(4,1 \%)$ que están en esta condición, frente a sus pares de las zonas rurales (24,1\%). En Lavalle en este aspecto, la diferencia es de 20 puntos, mientras que en la misma comparación sólo teniendo en cuenta los porcentajes provinciales es de 8 puntos (12,2\% en zonas urbanas y $20,0 \%$ en las rurales). De esta manera, la coexistencia de algunos factores como las responsabilidades de las tareas domésticas, junto al desfasaje del calendario escolar con el agrícola, incide en la permanencia en el sistema educativo y generan una alta tasa de deserción escolar. Con respecto a esto Cragnolino dirá que:

La coexistencia de asistencia escolar y trabajo doméstico -predial, extrapredial, remunerado o no- afecta el rendimiento educativo, determina el ausentismo esporádico o prolongado de los alumnos, y deriva, a veces, en el abandono definitivo de la escuela. Situación que se manifiesta con diferentes características según las regiones, pero que en general se inicia a los seis años de edad en diversas formas de trabajo doméstico, ocurre más abiertamente a partir de los diez u once años e implica entre los doce y catorce años la separación de la escuela y la incorporación plena a actividades laborales []. Bajo las condiciones descriptas, que devienen de la imposibilidad de conciliar calendario agrícola-ganadero, calendario escolar y las necesidades de subsistencia familiar, las alternativas que tiene el maestro para contrarrestar los efectos negativos de la situación no son muchas (Cragnolino, 2000:3). 


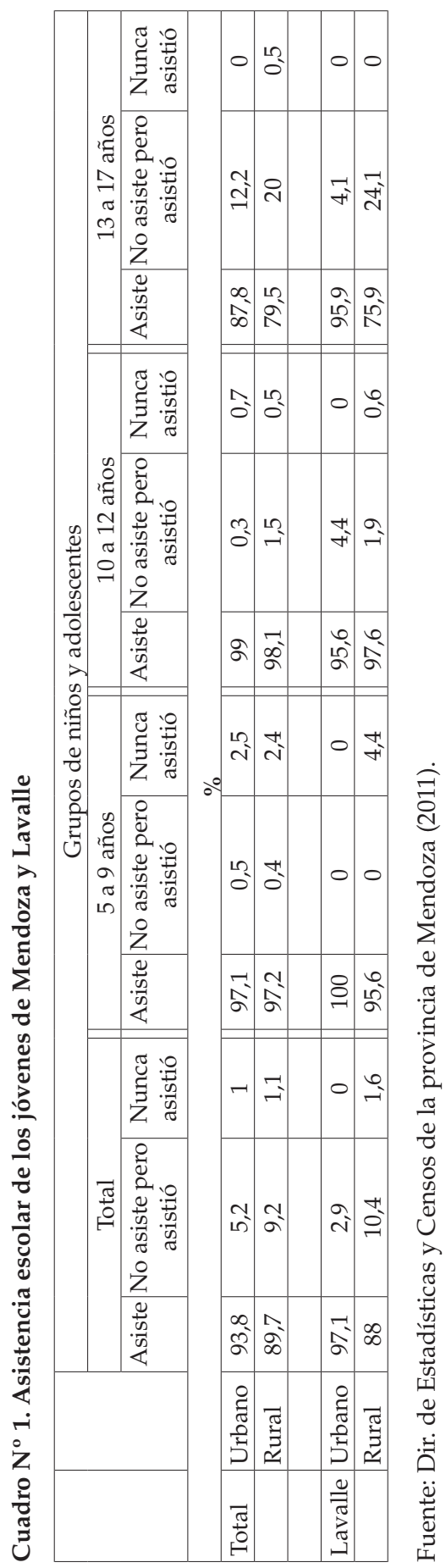


Tensiones entre ‘asistencia territorializada y ‘capacitación para la empleabilidad en espacios rurales: el caso de los jóvenes de Centro del Capacitación para el trabajo de Costa de Araujo, Mendoza (Argentina) - Diego Quattrini y Carla Rosales

El temprano contacto con el mundo del trabajo, muchas veces iniciado desde la infancia y profundizado en la adolescencia por las necesidades del ámbito familiar, culmina en algunos casos con la independencia económica. La misma es más permitida al grupo masculino, quien realiza tareas remuneradas, a diferencia del sector femenino, quien sólo logra su desprendimiento del grupo familiar cuando forma una nueva unidad doméstica por medio de la maternidad.

Todos los factores señalados en el apartado, cobran relevancia y complejización para entender el abandono y la deserción escolar en un medio rural fuertemente transformado y segmentado en cuanto a las condiciones materiales y sociales de sus pobladores. De allí que en Costa, emergieron en los últimos años circuitos educativos alternativos cargados de nuevas necesidades y demandas propias de una generación de jóvenes que deben enfrentar a un mundo con nuevas reglas de juego.

4. Los centros de Capacitación para el Trabajo: "Una asistencia territorializada"

Las mutaciones señaladas en este ámbito rural neo-colonial reconfiguraron no sólo las relaciones cotidianas dentro de la unidad doméstica, como las condiciones en el mundo agrario del trabajo, sino también, las formas de ofrecer protección social y movilidad para aquellos jóvenes empleados y desempleados de la región en situaciones de precariedad. En esta línea, la propuesta del trabajo tendrá el objetivo de analizar los modos de implementación de las políticas educativas y las prácticas que se despliegan a partir de su accionar, a fin más concreto de observar las maneras de intervenir sobre las sociabilidades, la soportabilidad y la aceptación social. Como estrategia se reflexionará sobre las lógicas de dominación a partir de la descripción de la propuesta educativa de re-escolarización (secundaria) dirigida a una franja de jóvenes pobladores rurales de Costa de Araujo que abandonaron el sistema de educación. 
Es difícil entender las políticas regionales actuales sin mencionar las consecuencias de sanción de la Ley Federal de Educación durante los años noventa en la Argentina. Esta ley significó un hito en la política pública educativa e introdujo cambios a partir de algunos ejes: cambios organizacionales (descentralización del sistema nacional educativo); curriculares y de contenido; y de estrategias políticas (Carranza, 2003). Este proceso de reconversión aún sigue vigente, ya que las leyes que se establecieron durante el último período poseen una cierta continuidad. Varios especialistas en educación coinciden en afirmar que la nueva ley nacional de educación promulgada en el 2007 conserva los aspectos liberales y no realiza crítica a los principios esenciales de su predecesora, como además sigue utilizando como sustento teórico los postulados de la teoría del capital humano (Romagnoli y Barreda, 2010:3)

La creación de los centros de capacitación para el trabajo (CCT) y la decisión de la administración provincial de los mismos se realizó en un contexto de mantener una estructura de descentralización organizacional y financiera educativa, en el que el Estado nacional sólo ha pretendido responsabilizarse en orientar contenidos y objetivos de la educación sin interpelar a la práctica concreta de las políticas educativas.

Los CCT son parte de un mosaico de ofertas educativas formales para jóvenes y adultos que el Estado cuenta para fomentar este vínculo educación - trabajo ${ }^{4}$. Su ejecución responde a una línea política de descentralización administrativa, financiera y de desarrollo territorial. Según datos de la Dirección Gene-

\footnotetext{
4 No es menor señalar, a pesar de la descentralización, la existencia de una cierta homogeneización en el plano enunciativo y normativo de las políticas educativas argentinas, las cuales apuntan a la necesidad de articular educación, calidad y competitividad ciudadana. Esta perspectiva educativa exige desarrollar en los individuos habilidades que los faculten para responder de manera efectiva a los imperativos de la globalización, es decir, para incrementar la productividad de las economías y la competitividad de las naciones.
} 
Tensiones entre ‘asistencia territorializada y ‘capacitación para la empleabilidad en espacios rurales: el caso de los jóvenes de Centro del Capacitación para el trabajo de Costa de Araujo, Mendoza (Argentina) - Diego Quattrini y Carla Rosales

ral de Escuela de Mendoza, la provincia posee hoy 80 Centros de Capacitación para el Trabajo distribuidos en sus distintos departamentos, de los cuales 4 se ubican en el departamento de Lavalle. La mayoría de los CCT no conservan una estructura fija de financiamiento, sino que al nacer al calor de la lógica de ajuste y de focalización producida en la educación, para mantenerse económicamente se ven obligados a buscar ofertas de políticas públicas disponibles que se presentan en los diferentes niveles de jurisdicción de gobierno (o en el sector privado). Muchas veces por este motivo se convierte a la figura del director como "un gestor de múltiples programas desarticulados", el cual debe mostrarse en alerta a las ofertas existentes, ser creativo para armar programas innovadores y trabajar sobre los requisitos, a fin de hacerse acreedor de distintos beneficios que poseen los planes disponibles. $\mathrm{Al}$ respecto la directora del CCT de Costa se mostraba orgullosa por haber "ganado en un concurso de proyectos socio-comunitarios 20 computadoras para la sala de informática", pero por otro lado lamentaba no contar con un presupuesto para solventar la merienda de sus alumnos. Contaba: "Los profesores colaboran para las tortitas, necesitamos solventar desde la escuela la comida, es una jornada de doble turno [] y muchos chicos vienen sin comer []. Hoy de eso están a cargo los docentes, ponen plata de su bolsillo, no les puedo pedir más nada a ellos" (Directora del CCT).

Estos centros (CCT), en Mendoza, al igual que los Centros de Educación Básica de adultos (CBA) y los Centros Educativos de Nivel Secundario (CENS), las ofertas están ajustadas a alumnos con edad extra-escolar, que provienen de fracasos escolares (por repeticiones, abandonos, embarazos, necesidad de trabajar, etc.). En este sentido, los CCT se muestran como salidas institucionales para aquellos alumnos que son considerados muchas veces en otros centros educativos como los "no capaces; no dotados, no disciplinados" (Romagnoli y Barreda, 2010: 12). 
La intervención de los CCT está enmarcada en una tarea de "asistencia territorializada" ${ }^{5}$. La misma se presenta como una oportunidad dirigida a un sector social específico que no tiene otra opción para escolarizarse. Para estos alumnos, no es el prestigio ni la calidad educativa la causa de la elección de estos tramos educativos, sino que la adhesión a estas escuelas es justificada por la posibilidad de concluir sus estudios. Una profesora del establecimiento hacía referencia a cómo la capacidad de elegir una escuela de acuerdo a su prestigio, es una estrategia de conservación y acumulación de bienes y conocimientos por parte de pocos sectores sociales, a la que los alumnos del CCT se encuentran al margen de desarrollarlas, resignándose a pertenecer a un segmento educativo devaluado:

Dentro del pueblo comparás [] el secundario técnico, es la escuela de prestigio, la de renombre []. Después hay otra escuela media que es la segunda, otra nueva que hay acá que está funcionando sólo los dos primeros años del secundario ( $8^{\circ}$ y 9 ) y esa es como más para abajo [] o sea para la mente de la comunidad, vos decís. ¿A dónde va a ir tu hijo a estudiar? Y no mi hijo va o al Instituto privado en Lavalle o a la técnica, y las otras ni, porque socialmente ya tienen esos prestigios... la gente le va dando esos valores, y con el CENS y los CCT ha pasado también lo mismo... siempre es el clásico: ah vas a CENS ahí te la regalan, ahí aprobás fácil, ahí no exigen nada [] no tenés que hacer nada. En la comunidad estaba instalada esa mirada (Docente del CCT).

5 El concepto de "asistencia territorializada" ha sido trabajado desde la preceptiva de la "focopolítica" desarrollada por Álvarez Leguizamon (2011:11): “La neutralización del conflicto o el control de la violencia se producen por medio de la descentralización a escala local y el autocontrol comunitario. Por oposición a la globalización desanclada, las políticas neoliberales y la focalización, territorializan e insularizan a los pobres rurales o urbanos, aunque algunos deben migrar porque la situación es insostenible". 
Tensiones entre ‘asistencia territorializada〉 y «capacitación para la empleabilidad en espacios rurales: el caso de los jóvenes de Centro del Capacitación para el trabajo de Costa de Araujo, Mendoza (Argentina) - Diego Quattrini y Carla Rosales

De algún modo, la oferta educativa territorial de los CCT colabora a consolidar dimensiones sociales de segregación en el habitar de la sociedad, es decir profundiza la fragmentación de los espacios geográficos, sociales y subjetivos de su población destinataria. El concepto de fragmentación, tal como es usado por Tiramonti (2008), marca la distancia entre distintos grupos sociales que reciben ofertas educativas. Bajo la actual lógica educativa y social, cada fragmento posee divergencias, que no sólo pueden medirse en términos de mayor o menor conocimiento, capitales culturales o habilidades intelectuales, sino que se distinguen además por convalidar pertenencias a mundos culturales diferentes entre sí en virtud de valores, expectativas y modos de vida que los organizan (Tiramonti, 2008:29).

5. Las intervenciones del CCT de Costa de Araujo: La terminalidad educativa - la educación para la empleabilidad.

El objetivo de este último apartado es analizar las intervenciones específicas de CCT de Costa de Araujo sobre los modos de sociabilidad, es decir sobre las formas de percepción y soportabilidad que desarrollan estos jóvenes. Para este propósito se contemplará su meta institucional de su oferta educativa: la formación en diferentes oficios y la teminalidad educativa.

El CCT trabaja coordinando esfuerzos con distintos organismos para promover planes de "terminalidad educativa exitosa"6. El proyecto de finalidad educativa se realiza principalmente a través de un convenio de articulación con el Centro de Educación Secundario (CENS) de la zona. En general los alumnos cursan en distintos horarios de tarde y de noche la terminalidad del secun-

6 Los planes de terminalidad educativa deben ser leídos en relación al mandato "obligatoriedad de la escuela secundaria" impulsada por la última ley federal de educación. Como resultado de esta obligatoriedad se promovieron un sinnúmero de programas de terminalidad nacional y provincial; algunos institucionalizados, como el caso del programa "de la esquina a la escuela" o los programas de capacitación para docentes de educación de jóvenes y adultos; y otros desarrollados en el marco de la gestión institucional de cada centro educativo. 
dario en las instalaciones del CCT y por la mañana aprenden un oficio.

La mayoría de los estudiantes que concurren al establecimiento proceden de familias que se insertan en la producción agrícola, en puestos de baja calificación. Una franja de los estudiantes "no estudian ni trabajan" (según la directora), en cambio otro grupo de alumnos trabajan principalmente en las distintas cosechas (tomate, aceituna, cebolla, uva), "podando, atando, cosechando" para distintas fincas, inclusive para las multinacionales de Cartellone y Molto. Sin embargo, varios profesores coinciden con que la mayoría de los jóvenes comenzaron a trabajar tempranamente y ya poseen experiencia laboral como trabajadores rurales: un docente comentaba:

Todos los chicos han trabajado... la mayoría seguro que ya ha trabajado alguna vez, no tienen el trabajo como algo ajeno, como algo que va a venir después de estudiar... ya vienen con experiencia laboral, como obreros rurales generalmente.

Los alumnos que cursan se localizan preferentemente en el rasgo de edad entre los 14 y los 26 años, aunque en la "terminalidad" cursan además de los jóvenes, algunos mayores. La escuela posee una población de 120 estudiantes. Muchos poseen como problema para asistir el transporte, debido a que provienen de zonas rurales alejadas y la escuela está ubicada en el centro del pueblo.

El CCT asumió como línea de trabajo preparar una oferta educativa dirigida a aquella franja de jóvenes que "no estudia ni trabaja". Esto hace que la escuela se posicione en un espacio institucional cuyo mandato social sea 'integrar' y "contener" a un conjunto de jóvenes que por su situación marginal serían representados como propensos a producir desbordes sociales. Cuenta la directora: 
Tensiones entre ‘asistencia territorializada〉 y «capacitación para la empleabilidad en espacios rurales: el caso de los jóvenes de Centro del Capacitación para el trabajo de Costa de Araujo, Mendoza (Argentina) - Diego Quattrini y Carla Rosales

Hace un par de años hicimos una encuesta en la zona, y nos dimos cuenta que había muchos chicos, que no hacían nada, estaban en la calle... como 200 jóvenes estaban fuera del sistema educativo y que tampoco trabajaban. Así comenzamos, a pesar de los retrasos administrativos y los problemas con el edifico, comenzamos a buscar una articulación con el CENS y el CEBA para generar una oferta para estos chicos.

Este segmento de jóvenes son calificados desde la institución bajo un diagnóstico en que se los asocia a categorías como "peligrosos" o "vagos". Y a partir de vincularlos subjetivamente estas marcas estigmatizantes justifican su intervención en cuanto mayor escolarización y encierro, en la necesidad de implementar mecanismos correctivos de control para identificar los riesgos y hacer que estos no se propaguen. La directora del CCT comentaba: "A mí me dicen que tengo cautiva una franja de población y de edad, pero yo digo que no, lo que buscamos es evitar la delincuencia que es una consecuencia de la exclusión de estos chicos". Esta representación de la juventud en las políticas públicas como sujetos en formación que necesitan ser regulados y encaminados es utilizada frecuentemente para argumentar e implementar una serie de políticas de acción del Estado, "compensatorias, focalizadas y de vigilancia", dirigidas a estas poblaciones. Así, bajo la relevancia que adquiere socialmente la justificación de la búsqueda de la cohesión social, aparece como trasfondo la producción de un proceso de regulación de su condición, en la que se criminaliza y se responsabiliza a muchos jóvenes por su situación de precariedad social.

La palabra contención aparece repetidamente en los objetivos del proceso educativo. "La escuela tiene la función de contención y de poder dar una segunda oportunidad, de poder ayudar a que lleguen a esa segunda oportunidad", comentaba una docente del CCT. De esta manera, esta lógica de control de la escuela se presenta al menos en un doble sentido: Primero, para mantener la matrícula efectiva de jóvenes escolarizados evitando la deserción. Y segundo, para brindar un soporte "afectivo" y ne- 
cesario a los alumnos, que no tuvieron en otros espacios. Sin este soporte difícilmente se logra prolongar la estadía en la escuela. Como se observa en Costa de Araujo, aparece una preocupación gubernamental por proponer ofertas educativas y pedagógicas diferenciales para el re-enganche exitoso de los alumnos pensado en función de la característica de estos jóvenes: un régimen asistencial y diferente en cuanto a las exigencias de contenido en las materias, en asistencias, programas que permiten cursar dos años escolares en uno solo, apoyos y extensión de plazos para rendir materias adeudas, etc. Una alumna que provenía de otro colegio y logró terminar el cursado en la institución contaba su experiencia:

Los profesores saben con los chicos que están tratando [] te entienden [] y más la preceptora que nos tocó era muy buena... saben que si llegás tarde es por algún motivo [], si estás mal o si te retrasás en una tarea, te dan tiempo ellos, porque saben que estás trabajando [] Yo pienso, si los chicos van ahí es por algún motivo [] sabían que si no entregabas la tarea a tiempo era porque estabas trabajando y no tuviste tiempo [] lo mismo para las pruebas, vos le decías: profe no pude estudiar y te decían: bueno [] y por ahí eso es más la comprensión de ellos.

De esta manera, la escuela cumple una cierta función asistencial; el sistema educativo aparece como un amortiguador del conflicto ante el incremento de la desigualdad. No logra resolver las asimetrías sino sólo compensa y "controla" a los desfavorecidos. A pesar de alcanzar importantes objetivos a la hora de escolarizar a la población, lo logra bajo la lógica de la asistencia fragmentada, y de profundizar circuitos educativos diferenciales en cuanto a los sistemas de aprendizaje, evaluación, acreditación y contenidos.

Esta fragmentación escolar también regula la auto-percepción de la capacidad del éxito de los alumnos. Las posibilidades de este sector social de elegir el "fragmento educativo" convalida una diferenciación en la adquisición de conocimientos, acti- 
Tensiones entre ‘asistencia territorializada) y ‘capacitación para la empleabilidad en espacios rurales: el caso de los jóvenes de Centro del Capacitación para el trabajo de Costa de Araujo, Mendoza (Argentina) - Diego Quattrini y Carla Rosales

tudes y expectativas de los alumnos de acuerdo a sus trayectorias disímiles en cuanto a las decepciones educativas, laborales y sociales. Aparecen frases como en "esta escuela (CCT) sí te comprenden los profesores cuando le decís que no pude estudiar y en las otras no" (alumna del CCT) o "me decidí de venir acá al Cens por cuestiones de que se me hacía más fácil" (alumna del CCT) En este caso, la oferta formativa del CCT de alguna manera limita a sus alumnos a alcanzar determinadas trayectorias posibles: al menos ofrece "poder terminar el colegio" o como mucho un "oficio certificado", "ser micro-emprendedor" o "vendedor de un local". Los límites que estas prácticas realizan en las "expectativas del sí mismo" en los alumnos termina funcionando como un dispositivo de regulación de las percepciones confirmadas inconscientemente a través de su experiencia educativa. Los jóvenes así quedan "calificados y autocalificados". Al respecto un docente ofrecía una observación sobre las expectativas de los alumnos:

Yo lo que he notado mucho es que les gusta eso [], conseguir un empleo [], el empleo ideal es en un local de venta de celulares [] que le pagan re poco [], yo he visto casos que le pagan 500 pesos al mes [] y los tienen trabajando entre 8 y 9 horas todos los días de lunes a sábado []; una alumna trabaja allí por 500 pesos, pero ella estaba contenta porque le daban plata por ir y estar en la onda de los celulares, vendiendo celulares y estando en un local (Docente del CCT).

La segunda meta institucional del CCT de Costa de Araujo es ofrecer programas de formación en oficios para mejorar las capacidades de las poblaciones marginales. En palabras de la directora: "Nuestra meta es calificar la mano de obra de Costa... Contamos con una amplia variedad de talleres, no sé, construcción, artesanías, pintura". Se asume la tarea de capacitar la fuerza de trabajo juvenil, partiendo de que a mayor certificación de educación que puedan proponer (tanto en una extensa variedad de oficios como en terminalidad educativa), provocaría una mejor 
empleabilidad, lo que mejoraría su "competitividad" en el mercado de trabajo de los estudiantes.

Los jóvenes que concurren pueden elegir para incrementar sus habilidades una variedad de ofertas de talleres y de aprendizajes en oficios (según publica el CCT en su folleto de propaganda hay cursos de gastronomía, bordado y tejido, artesanías en cuero, cerámica, modas, electricidad domiciliaria, gasista de $3^{a}$ categoría, construcción y plomería, y hasta de lengua portuguesa). Los talleres buscan instruir en oficios e impartir actitudes para el trabajo, es decir instaurar el modelo de la cultura del trabajo. Según la directora, "acá se plantea una tensión importante entre la asistencia social que reciben muchos y por otro lado lo que queremos apostar nosotros: la cultura del trabajo. Nosotros planteamos una educación integral".

Según el diagnóstico social que se realiza desde la escuela, el problema de la falta de oportunidades y la marginación sería la insuficiencia de calificación de los excluidos, y lo que se necesita habilitarlos en una formación que produzca en los individuos la obtención de aprendizajes que sean eficaces de acuerdo a los criterios del mercado:

Nosotros debemos cambiar la concepción de la escuela, en donde ya la producción no sólo es para la casa, sino que es para el mercado []. Por ejemplo, en Costa no pueden venir grandes empresas porque no hay mano de obra calificada (Directora de CCT de Costa de Araujo).

Educar para "capacitar" en estas condiciones, forma parte de una propuesta de la gestión gubernamental basada en la teoría del capital humano (Romagnoli et al., 2010; Álvarez Leguizamón, 2011). Los supuestos de la teoría corroboran que las causas de la precariedad social ("la falta de trabajo" diría la directora) se explica por la carencia de "capacidades individuales", siendo la pobreza un problema individual y no social, no entendiéndola como producto de las características de las relaciones sociales y las estructuras económicas de desigualdad. Así se fundamenta la 
Tensiones entre ‘asistencia territorializada) y (capacitación para la empleabilidad en espacios rurales: el caso de los jóvenes de Centro del Capacitación para el trabajo de Costa de Araujo, Mendoza (Argentina) - Diego Quattrini y Carla Rosales

necesidad de trabajar sobre la empleabilidad de los jóvenes, para mejorar y multiplicar sus capacidades de futuros trabajadores. La concepción educativa queda relacionada al desarrollo de "capitales", y a la adquisición de "activos personales" ${ }^{\prime 7}$, es decir una mezcla de habilidades, destrezas, valores y actitudes necesarios para vincularse al mundo del trabajo. Volverse ahora competente, involucra desarrollar actitudes flexibles y de responsabilidad que estén de acuerdo a las exigencias de cada sector productivo.

En esta línea, una de las apuestas del CCT es proyectar el aprendizaje en micro-emprendimientos. Aparece una educación que forma subjetividades en la autogestión y la cultura emprendedora e invita a desarrollar capacidades para que los alumnos generen sus propios negocios. Pero muchas veces, más que generar "nuevas empresas", refuerzan la subocupación y la autoexplotación de estas poblaciones. Además para que estas prácticas tengan éxito, los alumnos deberán alcanzar no sólo conocimientos específicos de determinados oficios, sino aprender a manejarse como si fuese una empresa y de responder actitudinalmente a las exigencias cambiantes y precarias que el mercado demanda. Y para lograr esto último existe una serie de valores que deberán incorporar, como el "esfuerzo, la perseverancia, el emprendimiento, la capacidad de resolver problemas", etc.

Lo que aparece en definitiva es la intención institucional de moderar una subjetividad que posibilite ciertas capacidades para la auto sustentabilidad. A pesar de la intención de apuntar a mejorar algunas habilidades de una franja de la población que proviene de fracasos educativos previos, se termina reforzando la formación de una fuerza de trabajo con una "empleabilidad

7 La idea de que todo lo "escaso" que estas poblaciones posean se convierta en "activos personales" o capital humano. De esta manera se pretende introducir la lógica de la valorización del mercado para aprobar y mercantilizar las relaciones de cotidianidad de las personas. Las viviendas precarias, los servicios básicos mínimos, el conocimiento y las relaciones de amistad se convierten ahora en patrimonio (Álvarez Leguizamón, 2011:8). 
diferencial", preparándola subjetiva y cognitivamente sólo para determinadas competencias demandadas y poco estimadas en un mercado cada vez más cambiante. El resultado puede tener tres caminos: la incorporación en los alumnos de capacidades que sirven para facilitar la obtención de trabajos en los periodos fluctuantes de desempleo, por el régimen de contratación y producción que caracteriza las actividades agrícolas; incorporación de habilidades necesarias para "venderse" junto con su emprendimiento en el incipiente mercado de servicios que posee el ámbito rural y así sobrevivir; y, el tercero, para los "no exitosos", la auto-culpabilización que promueve la aceptación social de las prácticas desiguales.

\section{Conclusiones}

Las mutaciones acontecidas en el capitalismo neocolonial no sólo se tradujo en la aplicación de nuevas modalidades de desposesión de los bienes naturales y de las energías de la fuerza de trabajo, sino en la irrupción sobre las mediaciones que conducen hacia formas socialmente adecuadas de sociabilidades. La actitud neocolonial puede ser observada en una variedad de dispositivos diseñados para los jóvenes - trabajadores. A lo largo del estudio se observó cómo la promoción de ciertas prácticas sociales, canalizadas en este caso desde un centro educativo, trabajan sobre las sensibilidades a fin alcanzar ciertos grados de aceptación social y de formar percepciones y capacidades en base a los lineamientos del mercado laboral. Estos dispositivos al territorializarse, en este caso en el espacio rural, reconfiguran de una manera diferencial relaciones sociales y formas de ver y entender el mundo.

Como alternativa de inclusión los CCT llevaron adelante una política pensada para jóvenes que presentan una deserción escolar temprana y dificultades para concluir sus estudios. Esta oferta educativa conduce a un camino educativo diferencial, tanto cognitivo, proponiendo un régimen de aprendizaje específico como subjetivo, preparando sociabilidades. En el caso específico 
Tensiones entre ‘asistencia territorializada〉 y «capacitación para la empleabilidad en espacios rurales: el caso de los jóvenes de Centro del Capacitación para el trabajo de Costa de Araujo, Mendoza (Argentina) - Diego Quattrini y Carla Rosales

del CCT de Costa de Araujo sus dos objetivos paradigmáticos -terminalidad educativa y capacitación en oficios- no deben ser leídos por fuera de las características de las políticas públicas oficiales, y que dan sentido a su existencia: contener afectivamente, regular las autopercepciones y las esperanzas de movilidad educativa y capacitar actitudinalmente a los alumnos. A través de sus metas institucionales, por un lado, el CCT se convierte en un medio de control cuyo fin es retener escolarmente a los jóvenes y desactivar la potencialidad de "peligrosos" que se les adjudica, y por otro incrementar una "empleabilidad diferencial" de su fuerza de trabajo, para ser usada en determinados ámbitos productivos, según las demandas del sector agrícola como del embrionario sector de servicio que presenta el mundo rural.

En definitiva, la existencia misma del CCT en un espacio agrario y periférico y su propuesta de promover las capacidades para un grupo social desafiliado, evidencia las modalidades que el campo político institucional posee para regular las maneras de pensar, sentir y proyectarse de los trabajadores. De ahí que la "lógica del emprendimiento" resume el contenido de los nuevos estilos de formación sobre las expectativas y los modos de vida de las poblaciones vulnerables. Proyectar la promesa en estos jóvenes de la inserción futura a partir del desarrollo de competencias personales para realizar micro-emprendimientos, es decir a través de la "autogestión y autoexplotación de sus capacidades", es animarlos y prepararlos a asumir los riesgos de enfrentar solos al mercado laboral, y de resignar a un segundo plano la promoción del trabajo colectivo y las potenciales vinculaciones sociales que culturalmente se encuentran presentes en estas comunidades.

\section{Bibliografía}

ÁLVAREZ LEGUIZAMÓN, Sonia (2011). Políticas sociales de transferencias condicionadas y cohesión social. En XXVIII Congreso Internacional da ALAS - Recife, 6 a 11 de setiembre de 2011. 
BENDINI, Mónica; RADONICH, Marta; STEIMBREGER, Norma (2007). Nuevos espacios agrícolas, mercado de trabajo y migraciones estacionales. En Radonich, Martha, Steimbreger, Norma y Giarraca, Norma (compiladoras). Reestructuraciones sociales en cadenas agroalimentarias. 1a ed. Buenos Aires: La Colmena.

CARRANZA, Alicia (2003). "Política y reforma educativa: los 'sentidos' posibles de los cambios", en Ortega, Facundo; Furlán, Alfredo; Carranza, Alicia; Hurtado, Carlos y Saleme de Burnichón, La educación hoy: una incertidumbre estructural. Córdoba: Brujas.

CAPUTO, Luis (2002). Informe de situación, juventud rural argentina 2000. Buenos Aires: Dirección Nacional de Juventud.

CRAGNOLINO, M. Elisa (2000). Ponencia: El alumno de la escuela rural y su condición de niño trabajador, II Congreso Internacional de Educación: "Debates y utopías". Facultad de Filosofía y Letras. UBA. Buenos Aires, 26 al 28 de julio del 2000. Ponente en la Comisión 84, "Diversidad y contexto cultural".

BOCCO, Adriana (2007). Transformaciones sociales y espaciales en la vitivinicultura mendocina. En Radonich, Martha, Steimbreger y Giarraca, Norma (compiladoras). Reestructuraciones sociales en cadenas agroalimentarias. 1a ed. Buenos Aires: La Colmena.

DURSTON, John (1998). "La situación de la juventud rural en América Latina. Invisibilidad y estereotipos". Santiago de Chile: CEPAL, Serie políticas sociales 28.

GUARANÁ DE CASTRO, Elisa (2009). Juventude rural no Brasil: procesos de exclusão e a construção de um ator político. En Revista Latinoamericana de Ciencia, Sociedad, Niñez y Juventud. N $^{\mathrm{o}}$ 7(1): 179-208. Manizales. Obtenido el 10 de febrero del 2012 de http:/ / www.umanizales.edu.co/revistacinde/index.html

KESSLER, Gabriel (2005). Estado del arte de la investigación sobre juventud rural en América Latina. Buenos Aires: EHESSUNGS.

MARGULIS, M. (2001). Juventud: una aproximación conceptual. En "Adolescencia y Juventud en América Latina". Burak, S. (Comp.). Costa Rica: Ed. LUR.

NEIMAN, Guillermo (2006). Los asalariados del campo en la Argenti- 
Tensiones entre ‘asistencia territorializada〉 y ‘capacitación para la empleabilidad en espacios rurales: el caso de los jóvenes de Centro del Capacitación para el trabajo de Costa de Araujo, Mendoza (Argentina) - Diego Quattrini y Carla Rosales

na: diagnóstico y políticas. 1a ed. Buenos Aires: Secretaría Agricultura, Ganadería, Pesca y Alimentación.

RODRÍGUEZ, Ernesto y DABEZIES, Bernardo (1991). Primer Informe sobre la juventud en América Latina 1990. Madrid. OIJ.

ROMAGNOLI, Cristina y BARREDA, Amelia (2010). Educación y reproducción de la desigualdad: políticas y prácticas educativas en el neoliberalismo. Obtenido el 30 de diciembre del 2010 de http:/ /bdigital.uncu.edu.ar/fichas.php?idobjeto=3191.

SCRIBANO, Adrián (2010). “TESIS 1: Colonia, Conocimiento(s) y Teorías Sociales del Sur", Onteaiken Boletín 10 (p. 1-22). Extraído el 10 de diciembre del 2010 desde http:/ / onteaiken. com.ar/ver/boletin10/0-1.pdf

SCRIBANO, Adrián (comp.) (2007a). Mapeando interiores. Cuerpo, conflicto y sensaciones. Córdoba, Jorge Sarmiento Editor, CEA-UNC.

TIRAMONTI, Guillermina (2008). Una aproximación a la dinámica de la fragmentación del sistema educativo argentino. Especificaciones teóricas y empíricas. En La Escuela Media en Debate. Problemas actuales y perspectivas desde la investigación. Tiramonti G. y Montes N. (comp.). Buenos Aires: Manantial Flacso. 